\title{
Is the Mean Platelet Volume a Predictive Factor for Atrial Fibrillation Developing After Coronary Artery Bypass Grafting in Elderly Patients?
}

\author{
Arda Aybars Pala, MD, Yusuf Salim Urcun, MD \\ Department of Cardiovascular Surgery, Adiyaman Training and Research Hospital, Adiyaman, Turkey
}

\section{ABSTRACT}

Background: Postoperative atrial fibrillation (PoAF) is the most common arrhythmic complication detected after coronary artery bypass grafting (CABG). It is associated with increased morbidity and mortality, especially in elderly patients. Mean platelet volume (MPV) shows the activation of platelets effective in the inflammatory and thrombotic process. The purpose of the present study was to investigate the relations between the preoperative MPV levels and development of PoAF in isolated CABG in elderly patients.

Methods: A total of 103 elderly patients (aged $\geq 65$ years), who underwent isolated $\mathrm{CABG}$ and were at preoperative sinus rhythm, were included in the study. Patients who did not have PoAF were identified as Group $1(\mathrm{~N}=74)$, and those with PoAF were identified as Group $2(\mathrm{~N}=29)$.

Results: PoAF incidence was 28.2\%. Preoperative MPV level was $8.41 \pm 1.13 \mathrm{fL}$ in Group 1 , and $9.28 \pm 1.00 \mathrm{fL}$ in Group 2. The difference was statistically significant $(P<.001)$. Multivariate logistic regression analysis revealed that age, preoperative hemoglobin, and preoperative MPV were independent predictive factors for PoAF development (OR [odds ratio]: $1.149,95 \%$ CI [confidence interval]: 1.043-1.265, $P=.005$; OR: $1.334,95 \%$ CI: $1.013-1.758, P=.040$; OR: $2.103,95 \%$ CI: $1.324-3.339, P=.002$, respectively). The cutoff value for MPV as the predictor of PoAF development was found to be 8.43 (sensitivity: $82.8 \%$ and specificity: $55.4 \%$ ).

Conclusion: This study showed that MPV levels are associated with PoAF development in elderly patients, and other independent predictive factors include age and preoperative hemoglobin levels for POAF development.

\section{INTRODUCTION}

At the present time, cardiovascular diseases are accepted as the most important cause of morbidity and mortality in the elderly population. The most important part of cardiovascular diseases in this respect consists of coronary artery diseases (CAD). Coronary artery bypass grafting (CABG), which is

Received fuly 18, 2020; accepted September 19, 2020.

Correspondence: Arda Aybars Pala, MD, Adiyaman Training and Research Hospital, Department of Cardiovascular Surgery, Yunus Emre, 1164. Street, 02200, Adiyaman, Turkey; +90-416-216-10-15; fax: +90-416-214-53-99 (e-mail:ardaaybars@hotmail.com). considered an effective method in CAD treatment, can be applied with a low morbidity and mortality level in the elderly population [Fink 2015]. Cardiac arrhythmias are common complications following CABG, and the most common cardiac arrhythmia after CABG (especially within 24-72 hours) is atrial fibrillation [Bohatch Júnior 2015]. Postoperative atrial fibrillation (PoAF) incidence was reported to be $20-40 \%$ [Anatoĺevna 2016]. Although PoAF is well-tolerated in most patients, it also causes hemodynamic disorder, thromboembolic events, and increased morbidity and mortality, especially in the elderly patient population [Luo 2017].

The pathophysiology of PoAF has not clearly been revealed today. It is known that the inflammation has a significant effect, and new risk factors are defined in this regard. One of these risk factors is the mean platelet volume (MPV), which is the indicator of the thrombocyte size and activation. When platelets, which are known to play important roles in inflammatory processes, are activated, their volumes become larger and their aggregate rates and prothrombotic potentials increase with metabolic and enzymatic activation [Kamath 2001]. Elevated MPV levels were considered to be indicative of inflammation and thrombosis and were associated

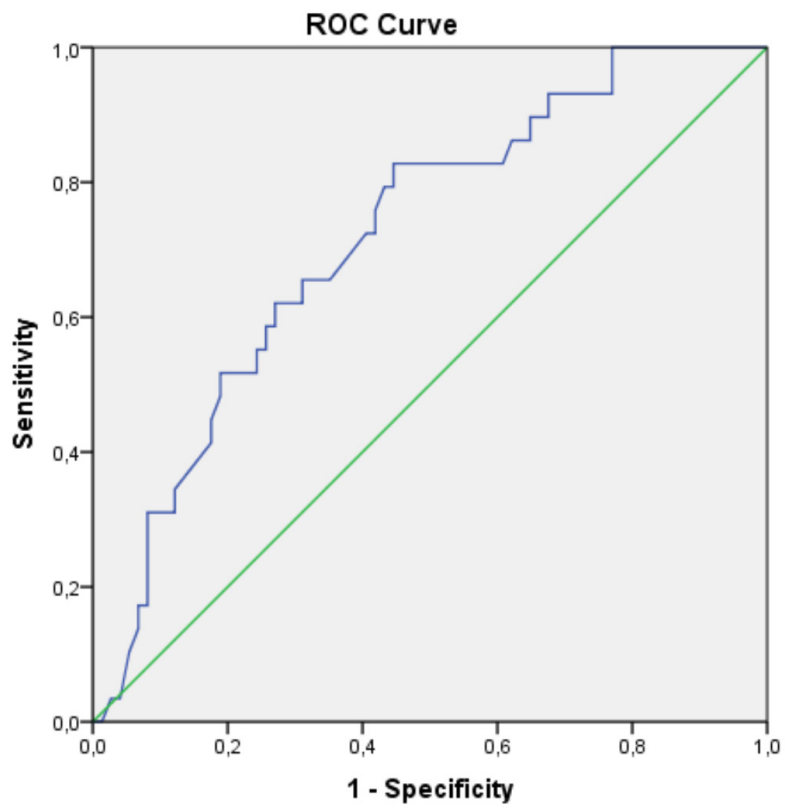

ROC curve of preoperative MPV for predicting to PoAF. 
Table 1. Preoperative data

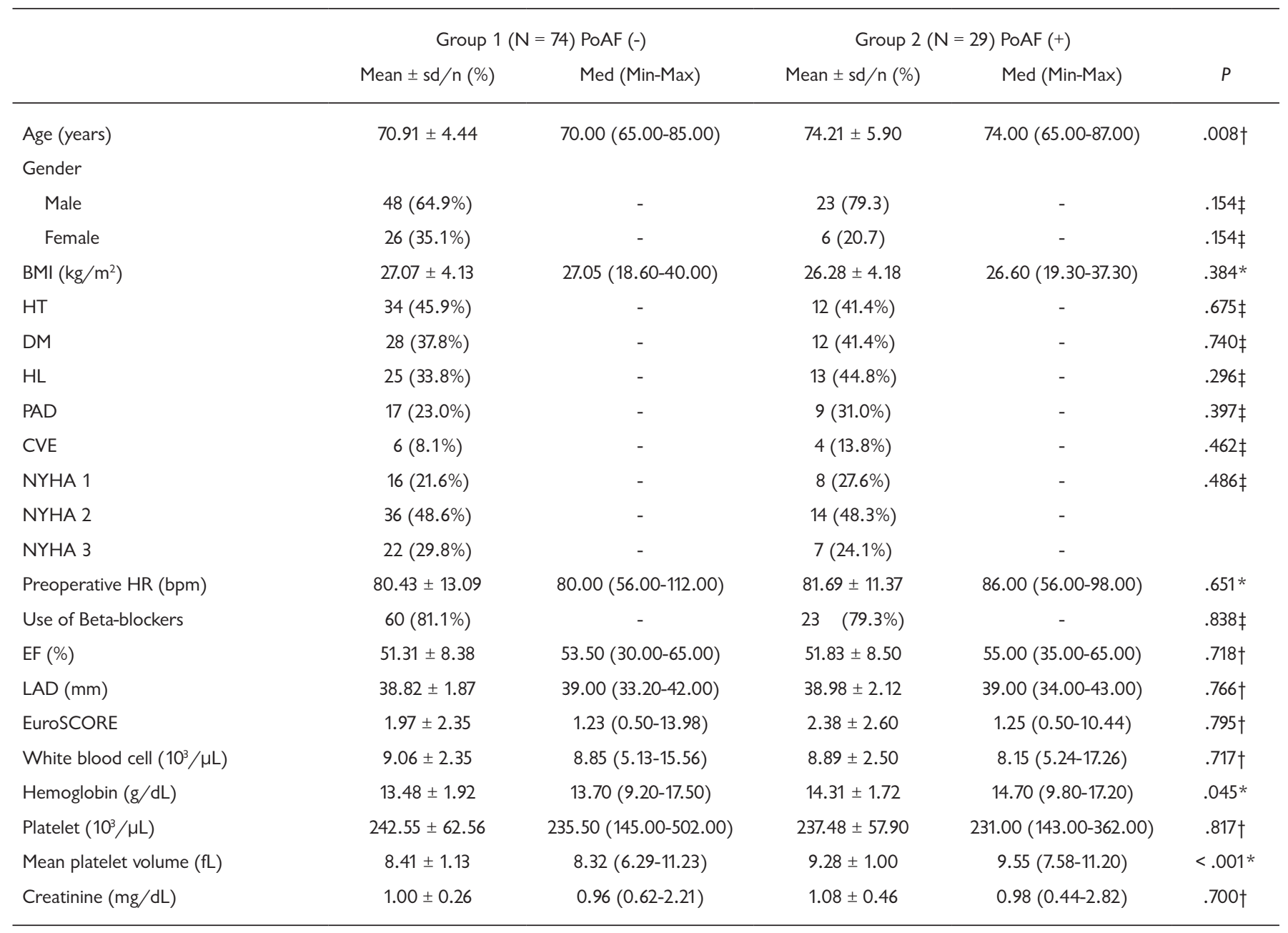

*Student-t test, †Mann-Whitney $U$ test, $\ddagger$ Chi-square test.

PoAF: postoperative atrial fibrillation, BMI: body mass index, HT: hypertension, DM: diabetes mellitus, HL: hyperlipidemia, PAD: peripheral artery disease, CVE: cerebrovascular events, NYHA: New York Heart Association functional class, HR: heart rate, EF: ejection fraction, LAD: left atrium diameter

with cerebrovascular and cardiovascular diseases [Xu 2015; Du 2016]. There are also several previous studies reporting that there is a relation between MPV and cardiac arrhythmia [Tekin 2013].

The purpose of the present study was to investigate whether preoperative MPV levels are predictors for PoAF, which is one of the factors that causes morbidity and mortality in elderly patients undergoing CABG.

\section{MATERIALS AND METHODS}

The data of the elderly patients who underwent isolated CABG at Adryaman Training and Research Hospital (Adiyaman, Turkey) Cardiovascular Surgery Clinic between January 2019 and March 2020 retrospectively were examined. A total of 103 patients aged 65 and over, who underwent isolated $\mathrm{CABG}$ with cardiopulmonary bypass $(\mathrm{CPB})$, were included in our study. Patient data were obtained from the hospital registration system, and local ethics committee approval was obtained (Approval number: 2020/5-27).

Patients at postoperative sinus rhythm were identified as Group $1(\mathrm{~N}=74)$, and patients with atrial fibrillation were identified as Group $2(\mathrm{~N}=29)$. Excluded from the study were patients who underwent emergent surgery, had previous CABG history, off-pump surgery, required additional surgical intervention except for CABG, had preoperative atrial fibrillation, were receiving preoperative amiodarone treatment, had chronic obstructive pulmonary disease (COPD), had chronic kidney or liver disease, acute or chronic infection, and any known malignancies or hematological problems.

Age, gender, body mass index, hypertension, diabetes mellitus, hyperlipidemia, peripheral artery disease, cerebrovascular event, New York Heart Association functional capacity 
Table 2. Perioperative and postoperative data

\begin{tabular}{|c|c|c|c|c|c|}
\hline & \multicolumn{2}{|c|}{ Group $1(\mathrm{~N}=74) \operatorname{PoAF}(-)$} & \multicolumn{2}{|c|}{ Group $2(\mathrm{~N}=29) \mathrm{PoAF}(+)$} & $P$ \\
\hline CPB time (min) & $102.22 \pm 29.01$ & $95.50(47.00-198.00)$ & $99.79 \pm 29.14$ & $100.00(30.00-163.00)$ & $.994 *$ \\
\hline Number of grafts used & $3.82 \pm 0.58$ & $4.00(2.00-5.00)$ & $3.76 \pm 0.83$ & $4.00(2.00-5.00)$ & $.883 *$ \\
\hline Drainage amount (cc) & $634.46 \pm 469.26$ & $500.00(100.00-2400.00)$ & $587.93 \pm 270.47$ & $600.00(200.00-1300.00)$ & $.610 *$ \\
\hline Length of stay in the ICU (day) & $1.42 \pm 0.97$ & $1.00(1.00-5.00)$ & $2.00 \pm 0.80$ & $2.00(1.00-5.00)$ & $<.001 *$ \\
\hline Length of hospital stay (day) & $6.16 \pm 1.53$ & $6.00(4.00-11.00)$ & $7.34 \pm 1.78$ & $7.00(4.00-12.00)$ & $.001 *$ \\
\hline
\end{tabular}

*Mann-Whitney $U$ test

PoAF: postoperative atrial fibrillation, CPB: cardiopulmonary bypass, ICU: intensive care unit.

classification, heart rate, beta-blocker drug use, ejection fraction, left atrium diameter, EuroSCORE, and preoperative laboratory parameters (white blood cell, hemoglobin, platelet, MPV, creatinine) were recorded as preoperative data. Patients with a blood pressure $\geq 140 / 90 \mathrm{mmHg}$ or antihypertensive drug use were considered as hypertension, fasting blood glucose $\geq$ $126 \mathrm{mg} / \mathrm{dL}$ or antidiabetic drug use were considered as diabetes mellitus, LDL cholesterol $\geq 130 \mathrm{mg} / \mathrm{dL}$, and total cholesterol $\geq$ $200 \mathrm{mg} / \mathrm{dL}$ or anti-hypertensidemic drug use were considered as hyperlipidemia. Heart rate was analyzed from preoperative 12-lead electrocardiography (ECG) records. Aortic crossclamp time, $\mathrm{CPB}$ time, number of grafts used, mechanical ventilation duration, drainage amount, length of stay in intensive care unit (ICU), and length of hospital stay were recorded as perioperative and postoperative data.

The blood samples taken from the antecubital vein after 12 hours of hunger during the preoperative period from the patients into EDTA (ethylenediaminetetraacetic acid) tubes were evaluated. The automatic blood counting device (Abbott CELL-DYN Ruby, Illinois, USA) was used for full blood count. The reference range for MPV was 6.8-10.8 fL in our hematology laboratory.

All patients were placed under general anesthesia and underwent a standard median sternotomy to reach the mediastinum. The saphenous vein grafts and left internal thoracic artery were prepared. CPB was performed by cannulating the ascending aorta and right atrium. Myocardial protection was applied to all patients by giving crystalloid cardioplegia and then cold blood cardioplegia. After giving warm blood cardioplegia, the aortic cross clamp was removed. A roller pump (non-pulsatile flow, $2.4 \mathrm{~L} / \mathrm{m}^{2} / \mathrm{min}$ ) and membrane oxygenator were used in all cases. Mean arterial pressure was maintained at $50-70 \mathrm{mmHg}$ during the aortic cross clamp, and hematocrit levels were 20-25\% during CPB. Moderate hypothermia $\left(30^{\circ} \mathrm{C}\right)$ was applied during aortic cross-clamp time, and the body was warmed to $36^{\circ} \mathrm{C}$ before weaning from CPB.

Patients continuously were monitored with five-lead telemetry in the ICU in the postoperative period. The patients were followed, in terms of heart rate, blood pressure measurement every four hours and 12-lead ECG recording every morning after discharge from the ICU. The patients were evaluated with 12-lead ECG recording, if heart rates increased in monitoring or in routine follow ups or if there were clinical arrhythmia signs (i.e. palpitation, shortness of breath, etc.). PoAF was diagnosed based on the ECG records. In PoAF treatment, amiodarone was applied in intravenous $300 \mathrm{mg}$ infusion in one hour, then intravenous $900 \mathrm{mg}$ infusion (maintenance dose) procedure in 24 hours. The patients were then administered with oral $200 \mathrm{mg}$ amiodarone three times a day.

The SPSS 11.5 Program was used in the analysis of the study data. Mean \pm standard deviation and median (minimummaximum) were used for quantitative variables as descriptive data, and number of patients (percent) was used for qualitative variables. In terms of quantitative variables, if there was a difference between the categories of qualitative variables with two categories, the Student-t test was used if the normal distribution assumption was met; and if not, the Mann-Whitney U-test was used. The Chi-Square and Fisher's Exact Test were used when the relations between the two qualitative variables were to be examined. The Receiver Operating Characteristic (ROC) analysis was performed to find a method to determine PoAF development for the quantitative variable, and the Youden Index value was used to calculate the cut-off value for the quantitative variable. Single and multi-variable logistics regression were employed to determine the risk factors affecting the PoAF development. Statistical significance was accepted at $P<.05$.

\section{RESULTS}

A total of 103 elderly patients were included in the present study, and PoAF developed at a rate of $28.2 \%(\mathrm{~N}=29)$. The demographic, clinical and laboratory findings of the patients are given in Table 1. The mean age of patients in Group 1 was found to be lower than in Group 2, and the difference was statistically significant $(70.91 \pm 4.44$ versus $74.21 \pm 5.90$, 
Table 3. Univariate logistic regression analysis for the possible predictors of PoAF

\begin{tabular}{|c|c|c|c|c|c|c|}
\hline Age & 0.128 & 0.045 & .005 & 1.136 & 1.040 & 1.241 \\
\hline DM & 0.148 & 0.447 & .740 & 1.160 & 0.483 & 2.784 \\
\hline $\mathrm{HL}$ & 0.465 & 0.447 & .298 & 1.592 & 0.663 & 3.825 \\
\hline $\mathrm{EF}$ & 0.008 & 0.027 & .777 & 1.008 & 0.956 & 1.061 \\
\hline LAD & 0.045 & 0.115 & .698 & 1.046 & 0.834 & 1.310 \\
\hline Preoperative WBC & 0.001 & 0.001 & .747 & 1.001 & 0.998 & 1.002 \\
\hline Preoperative $\mathrm{Hb}$ & 0.250 & 0.127 & .049 & 1.284 & 1.001 & 1.646 \\
\hline Preoperative Plt & -0.001 & 0.004 & .703 & 0.999 & 0.991 & 1.006 \\
\hline
\end{tabular}

B: beta coefficient, S.E.: standard error, OR: odds ratio, Cl: confidence interval, HT: hypertension, DM: diabetes mellitus, HL: hyperlipidemia, HR: heart rate, EF: ejection fraction, LAD: left atrium diameter, WBC: white blood cell, Hb: hemoglobin, Plt: platelet, MPV: mean platelet volume, Cr: creatinine

$P=.008)$. The patient groups were similar, in terms of demographic data and clinical characteristics except for age.

When the laboratory parameters were compared, preoperative hemoglobin levels were $13.48 \pm 1.92 \mathrm{~g} / \mathrm{dL}$ in Group 1 , and $14.31 \pm 1.72 \mathrm{~g} / \mathrm{dL}$ in Group 2 . The difference was statistically significant $(P=.045)$. Preoperative MPV level was 8.41 $\pm 1.13 \mathrm{fL}$ in Group 1, and $9.28 \pm 1.00 \mathrm{fL}$ in Group 2. The elevated preoperative MPV level in Group 2 was statistically significant $(P<.001)$. In addition, preoperative white blood cell, platelet, and creatinine levels did not differ at significant levels between the groups.

Perioperative variables did not differ at significant levels between the groups, which showed that similar surgical procedures were performed in similar periods. When postoperative data were compared, there were no significant differences between the groups in terms of mechanical ventilation duration and drainage amounts. Compared with Group 2, patients in Group 1 had significantly lower length of stay in the ICU $(1.42 \pm 0.97$ days versus $2.00 \pm 0.80$ days, $P<.001)$ and length of hospital stay $(6.16 \pm 1.53$ days versus $7.34 \pm 1.78$ days, $P=.001)$ (Table 2).

Univariate logistic regression analysis was made to evaluate any possible predictors in PoAF development (Table 3). It was detected that age, preoperative hemoglobin, and preoperative MPV were significantly related with PoAF development in elderly patients. As a result of the univariate logistic regression analysis, statistically significant variables were included in the multivariate logistic regression analysis. As a result of the multivariate logistic regression analysis, age (OR [odds ratio]: $1.149,95 \%$ CI [confidence interval]: $1.043-1.265, P=.005)$, preoperative hemoglobin (OR: 1.334, 95\% CI: 1.013-1.758, $P=.040)$ and preoperative MPV (OR: 2.103, 95\% CI: $1.324-$ $3.339, P=.002$ ) were shown to be independent predictive factors in elderly patients for PoAF development (Table 4).
ROC curve analysis was made to determine the predictive effect of MPV levels in PoAF development in elderly patients. The area under the curve was 0.719 for MPV (95\% CI: $0.614-$ $0.824, P=.001)$. The ideal cut-off value for MPV was found to be 8.43 , according to Youden Index (sensitivity: $82.8 \%$ and specificity: $55.4 \%$ ) (Figure).

\section{DISCUSSION}

In our study, we found that the elevated preoperative MPV levels have a significant relation with PoAF, which develops in elderly patients who underwent isolated CABG. We also aimed to determine the predictors for PoAF development, and in the multivariate logistic regression analysis, we found that age, preoperative hemoglobin and preoperative MPV were independent predictive factors. One unit increase in the quantitative variable of age value increased PoAF development risk 1.149 times, one unit increase in the quantitative variable of preoperative hemoglobin value increased PoAF development risk 1.334 times, and one unit increase in the quantitative variable of preoperative MPV value increased PoAF development risk 2.103 times.

In our present day, as a result of increasing life expectancy and improvements in cardiac surgery, CABG can be applied safely in elderly patients. However, since the cardiac reserves of this patient group are more limited, it is necessary to be careful, in terms of complications that might occur during the postoperative period. Knowing the predictive factors of atrial fibrillation, which is the most common cardiac arrhythmia after $\mathrm{CABG}$, is important in this respect.

PoAF incidence was reported to be 20-40\% [Anatoĺevna 2016]. In our study, it was found that PoAF was $28.2 \%$ in elderly patients with isolated CABG. In addition, in our study, 
Table 4. Multivariate logistic regression analysis for the possible predictors of PoAF

\begin{tabular}{|c|c|c|c|c|c|c|}
\hline Age & 0.139 & 0.049 & .005 & 1.149 & 1.043 & 1.265 \\
\hline Preoperative MPV & 0.743 & 0.236 & .002 & 2.103 & 1.324 & 3.339 \\
\hline
\end{tabular}

B: beta coefficient, S.E.: standard error, OR: odds ratio, $\mathrm{Cl}$ : confidence interval, $\mathrm{Hb}$ : hemoglobin, MPV: mean platelet volume

it was found that the PoAF developing patient group was significantly older than the patient group that did not develop PoAF. It already is known that advanced age causes atrial fibrillation by causing atrial anatomical and electrophysiological changes [Allessie 2001]. There are studies showing that advanced age is an important predictor for PoAF [Ozsin2018; Mathew 2004]. Nisanoglu et al included 426 elderly patients in their study (age > 65) and reported that advanced age was a predictor for the development of post-CABG atrial fibrillation (OR: $1.07, P=.009$ for age $>65$; and OR: $1.77, P=.042$ for age $>75$ ) [Nisanoglu 2007]. Similar to the studies in the literature, our study also found in univariate and multivariate logistic regression analysis that age is significantly associated with PoAF development.

It already is known that inflammation is an important factor in PoAF pathophysiology. It is considered that atrial fibrillation develops as a result of the inflammatory mediators and immune cells in inflammatory response affecting the cardiac tissue [Hu 2015]. Endothelial dysfunction and vascular inflammation associated with it are blamed in the pathogenesis of many chronic diseases. More frequent atrial fibrillation is observed in chronic diseases because of this inflammation [Grover-Paez 2009; Khan 2020]. Mathew et al conducted a study in which they included 4657 CABG patients and reported that there was $43 \%$ increase in the probability of PoAF development in the presence of COPD (OR: 1.43, 95\% CI: $1.09-1.87, P=.009)$ [Mathew 2004]. There are studies reporting a significant relation between preoperative chronic renal failure and PoAF development [Nisanoglu 2007; Auer 2007]. It also was reported that the incidence of atrial fibrillation is high in chronic liver diseases [Huang 2018]. For these reasons, COPD, chronic kidney and liver disease patients were excluded from our study. In addition, the lack of significant difference between the groups, in terms of risk factors that have inflammation in their pathogenesis like hypertension, diabetes mellitus, hyperlipidemia, peripheral artery disease and cerebrovascular event, increases the reliability of the results of our study.

One of the routine tests applied in the preoperative period in patients with CABG is the full blood count test. This test, which is sensitive to the hemodynamic changes in the body, contains parameters that have predictive value for PoAF [Weymann 2018]. Among these hematological parameters, the relation of MPV with PoAF is remarkable. MPV is the indicator of platelet size, and there is a relation between the function and size of platelets. Young and large-volume platelets produced in inflammatory processes are more active both in enzymatic and metabolic terms. Many inflammations, coagulation and thrombosis mediators in the body are released by these large platelets [Gasparyan 2011]. For this reason, studies were conducted in the past to investigate the relations between PoAF and MPV, which are known to have an effect of inflammation in pathophysiology. Erdem et al conducted a study to evaluate 208 elective isolated CABG patients and found that MPV was an independent predictor for PoAF development (OR: 2.564, 95\% CI: 1.326-4.958, $P=.005$ ) [Erdem 2014]. In another study, Şaşkın et al evaluated 1138 patients who underwent isolated CABG and had preoperative normal sinus rhythm. Preoperative MPV levels were found to be significantly higher in the PoAF developing group compared with the group with postoperative normal sinus rhythm $(9.03 \pm 0.74 \mathrm{fL}$ versus $8.32 \pm 0.60 \mathrm{fL}, P=.0001)$. They found that the MPV cut-off level for PoAF development as $8.65 \mathrm{fL}$ (sensitivity: $74.1 \%$ and specificity: $68.4 \%$ ) [Şaşkın 2016]. In a systemic review conducted with metaanalysis, Weymann et al reported that MPV was the definitive predictor of PoAF development and recommended its inclusion in atrial fibrillation diagnostic criteria [Weymann 2018]. In our study, our results were in line with the literature data. The preoperative MPV levels were found to be significantly higher in our group, in which we included patients developing PoAF in elderly patients undergoing isolated CABG. In the multivariate logistic regression analysis, it was found that the preoperative MPV levels were an independent predictive factor for PoAF development.

In many previous studies, it was reported that there is a relation between preoperative low hemoglobin levels and postoperative negative events, such as atrial fibrillation in cardiac surgery [Bell 2008; Miceli 2014]. Unlike these studies, although the mean preoperative hemoglobin levels of our patient group that developed PoAF were found to be at slightly higher levels in our study compared with the patient group without PoAF development, this difference was statistically significant. In addition, the preoperative hemoglobin level was found to be an independent predictive factor for PoAF.

It already is known that CPB is associated with PoAF development as a cause of systemic inflammatory response [Narducci 2014]. The fact that there were no significant differences between the perioperative variables in our study may be considered to indicate that CPB did not affect our results. In addition, length of stay in the ICU and length of hospital 
stay, which were among postoperative variables evaluated in our study, being significantly higher in PoAF group can be explained by the hemodynamic instability and the need for additional treatment due to PoAF.

Our study had some limitations. It was a retrospective study, and a limited number of patients were included because it had a single-center design. In addition, only the cardiac rhythms of the patients until the patients were discharged from the hospital were monitored and evaluated. One thing that stood out in our study, which included elderly patients, was that both age and MPV levels were significantly higher in our group patients who developed PoAF. Previous studies reported that MPV levels increase with advanced age [Verdoia 2015; Lippi 2012]. In our study, which included patients of advanced ages, we associated the elevated MPV levels in our PoAF developing patient group with the underlying inflammation. More comprehensive studies that will be conducted on age, MPV and PoAF development are needed to clarify this point.

\section{CONCLUSION}

According to the data obtained in our study, there is a relation between high preoperative MPV levels and PoAF development after CABG, in elderly patients. Our analysis showed that age, preoperative hemoglobin and preoperative MPV levels are independent predictive factors for PoAF development after isolated CABG in elderly patients.

\section{REFERENCES}

Allessie MA, Boyden PA, Camm AJ, et al. 2001. Pathophysiology and prevention of atrial fibrillation. Circulation 103(5): 769-77.

Anatoĺevna RO, Veniaminovich FO, Mikhaylovich KS. 2016. Predictors of new-onset atrial fibrillation in elderly patients with coronary artery disease after coronary artery bypass graft. J Geriatr Cardiol 13(5): 444-9.

Auer J, Lamm G, Weber T, et al. 2007. Renal function is associated with risk of atrial fibrillation after cardiac surgery. Can J Cardiol 23(11): 859-63.

Bell ML, Grunwald GK, Baltz JH, et al. 2008. Does preoperative hemoglobin independently predict short-term outcomes after coronary artery bypass graft surgery? Ann Thorac Surg 86(5): 1415-23.

Bohatch Júnior MS, Matkovski PD, Di Giovanni FJ, Fenili R, Varella EL, Dietrich A. 2015. Incidence of postoperative atrial fibrillation in patients undergoing on-pump and off-pump coronary artery bypass grafting. Rev Bras Cir Cardiovasc 30(3): 316-24.

Du J, Wang Q, He B, et al. 2016. Association of mean platelet volume and platelet count with the development and prognosis of ischemic and hemorrhagic stroke. Int J Lab Hematol. 38(3): 233-9.

Erdem K, Ayhan S, Ozturk S, et al. 2014. Usefulness of the mean platelet volume for predicting new-onset atrial fibrillation after isolated coronary artery bypass grafting. Platelets 25: 23-6.

Fink HA, Hemmy LS, MacDonald R, et al. 2015. Intermediate- and Long-Term Cognitive Outcomes After Cardiovascular Procedures in
Older Adults: A Systematic Review. Ann Intern Med 163(2): 107-17

Gasparyan AY, Ayvazyan L, Mikhailidis DP, Kitas GD. 2011. Mean platelet volume: a link between thrombosis and inflammation? Curr Pharm Des 17(1): 47-58.

Grover-Paez F, Zavalza-Gomez AB. 2009. Endothelial dysfunction and cardiovascular risk factors. Diabetes Res Clin Pract 84(1): 1-10.

Hu YF, Chen YJ, Lin YJ, Chen SA. 2015. Inflammation and the pathogenesis of atrial fibrillation. Nat Rev Cardiol 12(4): 230-43.

Huang WA, Dunipace EA, Sorg JM, Vaseghi M. 2018. Liver disease as a predictor of new-onset atrial fibrillation. J Am Heart Asso 7(15): $\mathrm{e} 008703$.

Kamath S, Blann AD, Lip GY. 2001. Platelet activation: assessment and quantification. Eur Heart J 22(17): 1561-71.

Khan AA, Thomas GN, Lip GYH, Shantsila A. 2020. Endothelial function in patients with atrial fibrillation. Ann Med 52(1-2): 1-11.

Lippi G, Meschi T, Borghi L. 2012. Mean platelet volume increases with aging in a large population study. Thromb Res 129(4): e159-60.

Luo W, Huaibin W, Wenjun Z, et al. 2017. Predictors of postoperative atrial fibrillation after isolated on-pump coronary artery bypass grafting in patients $\geq 60$ years old. Heart Surg Forum. 20(1): E038-E042.

Mathew JP, Fontes ML, Tudor IC, et al. 2004. A multicenter risk index for atrial fibrillation after cardiac surgery. JAMA 291(14): 1720-9.

Miceli A, Romeo F, Glauber M, de Siena PM, Caputo M, Angelini GD. 2014. Preoperative anemia increases mortality and postoperative morbidity after cardiac surgery. J Cardiothorac Surg 9: 137.

Narducci ML, Pelargonio G, Rio T, et al. 2014. Predictors of postoperative atrial fibrillation in patients with coronary artery disease undergoing cardiopulmonary bypass: a possible role for myocardial ischemia and atrial inflammation. J Cardiothorac Vasc Anesth 28(3): 512-9.

Nisanoglu V, Erdil N, Aldemir M, et al. 2007. Atrial fibrillation after coronary artery bypass grafting in elderly patients: incidence and risk factor analysis. Thorac Cardiovasc Surg 55(1): 32-8.

Ozsin KK, Sanri US, Toktas F, Yavuz S. 2018. Relationship between red cell distribution width and mean platelet volume with new onset atrial fibrillation after off-pump coronary artery bypass grafting. Bratisl Lek Listy 119(6): 335-340.

Şaşkın H, Düzyol Ç, Aksoy R, Özcan KS, Güngör B, İdiz M. 2016. Do preoperative C-reactive protein and mean platelet volume levels predict development of postoperative atrial fibrillation in patients undergoing isolated coronary artery bypass grafting? Postepy Kardiol Interwencyjnej 12(2): 156-63

Tekin G, Tekin YK, Sivri N, Yetkin E. 2013. Mean platelet volume in patients with nonvalvular atrial fibrillation. Blood Coagul Fibrinolysis 24(5): 537-9.

Verdoia M, Schaffer A, Barbieri L, et al. 2015. Impact of age on mean platelet volume and its relationship with coronary artery disease: a singlecentre cohort study. Exp Gerontol 62: 32-6.

Weymann A, Ali-Hasan-Al-Saegh S, Popov AF, et al. 2018. Haematological indices as predictors of atrial fibrillation following isolated coronary artery bypass grafting, valvular surgery, or combined procedures: a systematic review with meta-analysis. Kardiol Pol 76(1): 107-118.

Xu XF, Jiang FL, Ou MJ, Zhang ZH. 2015. The association between mean platelet volume and chronic atrial fibrillation and the presence of thrombotic events. Biomed Rep 3(3): 388-394. 